УДК 542.943-92’78

\title{
Parameters for Quantitative Evaluation of the Radical-Generating (Pro-Oxidant) Capacity of Metal Ions and the Radical-Scavenging Activity of Antioxidants Using Voltammetric Method
}

Valentina N. Batalova, Yurii G. Slizhov and Anton A. Chumakov* National Research Tomsk State University 36 Lenin Str., Tomsk, 634050, Russia

Received 10.08.2015, received in revised form 16.12.2015, accepted 04.03.2016

In the system, which combines the Fenton reaction or similar (of iron, cobalt, nickel or copper ions with hydrogen peroxide) with the registration of the hydroxyl radical cathodic reduction voltammetric current, the parameters were adjusted for quantitative evaluation of the radical-generating (prooxidant) capacity of metal ions and activity of antioxidants in radical stress induced by metal ions of different nature. Measure of metal ions pro-oxidant activity is the value of the regression coefficient (expressed in plus microAmpere per micromol) of equation for linear approximation, constructed in the coordinates of analytical signal increasing relative to metal ions number in the reaction system. The value of the same regression coefficient (expressed in minus microAmpere per micromol) of equation for linear approximation, constructed in the coordinates of analytical signal decreasing relative to the amount of antioxidant (ascorbic acid) in the reaction system, is a measure of the antioxidant activity of substance.

Keywords: iron, cobalt, nickel, copper, Fenton reaction, hydroxyl radical, voltammetry, prooxidants, antioxidants, ascorbic acid.

DOI: 10.17516/1998-2836-2016-9-1-60-67.

(C) Siberian Federal University. All rights reserved

* Corresponding author E-mail address: anton.doktor.tomsk@mail.ru 


\title{
Параметры количественной оценки
}

\author{
радикал-генерирующей (прооксидантной) способности \\ ионов металлов и антирадикальной \\ активности антиоксидантов \\ при использовании вольтамперометрического метода
}

\author{
В.Н. Баталова, Ю.Г. Слижов, А.А. Чумаков \\ Наииональный исследовательский \\ Томский государственный университет \\ Россия, 634050, Томск, пр. Ленина, 36
}

\begin{abstract}
В системе, комбинирующей реакцию Фентона или подобную ей (ионов железа, кобальта, никеля или меди с пероксидом водорода) с вольтамперометрической регистрацией тока катодного восстановления гидроксильного радикала, определены параметры количественной оценки радикал-генерирующей (прооксидантной) способности ионов металлов и активности антиоксидантов при радикальном стрессе, индуцированном ионами металлов разной природы. Мерой прооксидантной активности ионов металла является значение коэффициента регрессии (выраженное в плюсмикроАмпер-на-микромоль) уравнениялинейной аппроксимации, построенной в координатах прироста величины аналитического сигнала относительно количества в реакционной системе ионов металла. Значение аналогичного коэффициента регрессии (выраженное в минус микроАмпер-на-микромоль) уравнения линейной аппроксимации, построенной в координатах убыли величины аналитического сигнала относительно количества антиоксиданта (аскорбиновой кислоты) в реакционной системе, служит мерой антиоксидантной активности вещества.
\end{abstract}

Ключевые слова: железо, кобальт, никель, медь, реакция Фентона, гидроксильный радикал, вольтамперометрия, прооксиданты, антиоксиданты, аскорбиновая кислота.

\section{Введение}

Роль свободнорадикальных реакций в биологических системах, в частности в процессах химического канцерогенеза и старения, концепция антиоксидантов как «гасителей» свободных радикалов (free radical scavengers) и средств для профилактики рака и преждевременного старения подробно обсуждены ещё в 70-80-х гг. XX в [1]. Роль ионов железа и меди как инициаторов и катализаторов радикальных реакций и медиаторов окислительных повреждений в организме стали дискутировать позднее [2, 3]. С 1990-х гг. активно исследуют процессы генерирования в биологических системах супероксидного, гидроксильного радикалов и других активных форм кислорода ионами металлов переменной валентности в окислительновосстановительных реакциях. К настоящему времени сформулирована патогенетическая концепция металл-индуцированного окислительного стресса в биологических системах, 
возникающего в результате нарушений гомеостаза ионов металлов и приводящего к повреждениям нуклеиновых кислот, перекисному окислению липидов, модификациям белков и другим эффектам, лежащим в основе сердечно-сосудистых, онкологических заболеваний, диабета и другой патологии $[4,5]$.

Исследования радикал-генерирующей (прооксидантной) способности ионов металлов различной природы и зависимости активности антиоксидантов от природы ионов металлов, провоцирующих окислительный стресс, являются актуальными.

Целью нашей работы было определение параметров количественной оценки прооксидантной способности ионов металлов в модельных реакциях генерирования гидроксильного радикала и антирадикальной активности антиоксидантов при использовании вольтамперометрического метода регистрации гидроксильного радикала.

\section{Экспериментальная часть}

Bещества: растворы пероксида водорода, железа(II) сульфата, кобальта(II) сульфата, никеля(II) сульфата, меди(II) сульфата, аскорбиновой кислоты. Все использованные реактивы относились к категории химически чистых.

Модельные реакции. Воспроизводились реакции генерирования гидроксильного радикала $\mathrm{HO}^{`}$ из $\mathrm{H}_{2} \mathrm{O}_{2}$ ионами металлов переменной валентности - классическая реакция Фентона $\left(\mathrm{Fe}^{2+} / \mathrm{H}_{2} \mathrm{O}_{2}\right)$ и подобные ей $[6,7]$.

Аналитическая методика. Использовался вольтамперометрический сигнал (ток катодного восстановления) гидроксильного радикала [8]. Электрохимическая ячейка (10 мл) заполнялась фоновым 0,1 М раствором сульфата натрия, добавлялось 5,00 мкмоль пероксида водорода. Анализатор СТА-1 (ООО «ИТМ», г. Томск), совмещённый с компьютером. Рабочий электрод ртутно-плёночный на серебряной подложке, электрод сравнения хлорид-серебряный. Анализ проводился в режиме постояннотоковой вольтамперометрии с развёрткой потенциала в катодную область от 0 до -2 В со скоростью 40 мВ/с.

Оценка прооксидантной способности ионов металлов. В электрохимической ячейке постепенно увеличивалось абсолютное содержание ионов $\mathrm{Fe}^{2+}, \mathrm{Co}^{2+}, \mathrm{Ni}^{2+}$ или $\mathrm{Cu}^{2+}$ от 0,50 до 3,50 мкмоль путём порционного добавления раствора сульфата исследуемого металла, регистрировалась динамика силы тока катодного восстановления гидроксильного радикала I, мкА (рис. 1). Проводился корреляционно-регрессионный анализ (Microsoft Excel) зависимостей $\mathrm{I}=f\left(\mathrm{nFe}^{2+}\right)$, $\mathrm{I}=f\left(\mathrm{nCo}^{2+}\right), \mathrm{I}=f\left(\mathrm{nNi}^{2+}\right), \mathrm{I}=f\left(\mathrm{nCu}^{2+}\right)$, где $\mathrm{n}-$ абсолютное содержание ионов металла в ячейке (мкмоль), результаты представлены на рис. 2.

Оценка антиоксидантной активности аскорбиновой кислоты. В электрохимическую ячейку вносилось 3,50 мкмоль $\mathrm{Fe}^{2+}, \mathrm{Co}^{2+}, \mathrm{Ni}^{2+}$ или $\mathrm{Cu}^{2+}$, регистрировалась $\mathrm{I}_{0}$, далее в ячейке постепенно увеличивалось абсолютное содержание аскорбиновой кислоты, nAK, от 0,50 до 20,00 мкмоль. Проводился корреляционно-регрессионный анализ (Microsoft Excel) зависимостей $\mathrm{I}=f(\mathrm{nAK})$ для каждой серии, результаты представлены на рис. 3.

\section{Обсуждение результатов}

Оценка прооксидантной способности ионов металлов. Согласно полученным результатам (рис. 2) сила тока катодного восстановления гидроксильного радикала линейно коррелиру- 

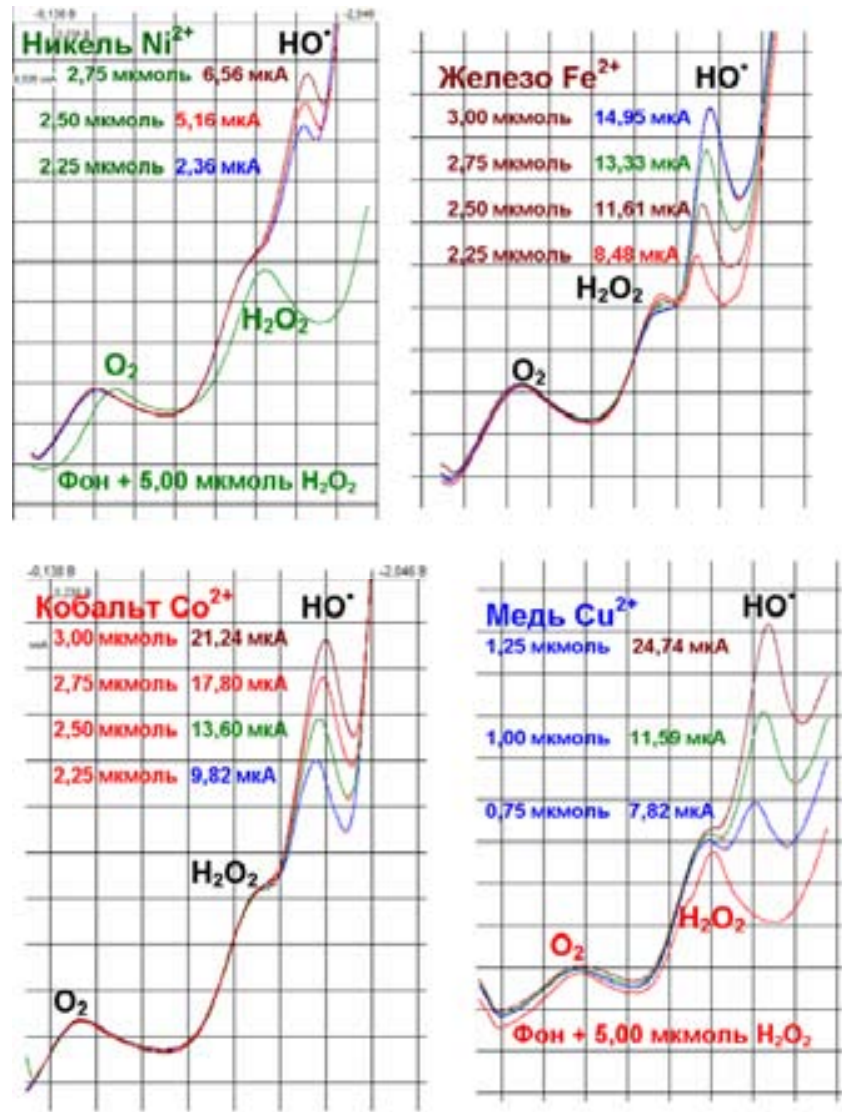

Рис. 1. Вольтамперограммы в $0,1 \mathrm{M}$ растворе $\mathrm{Na}_{2} \mathrm{SO}_{4}$ с добавками ионов металлов

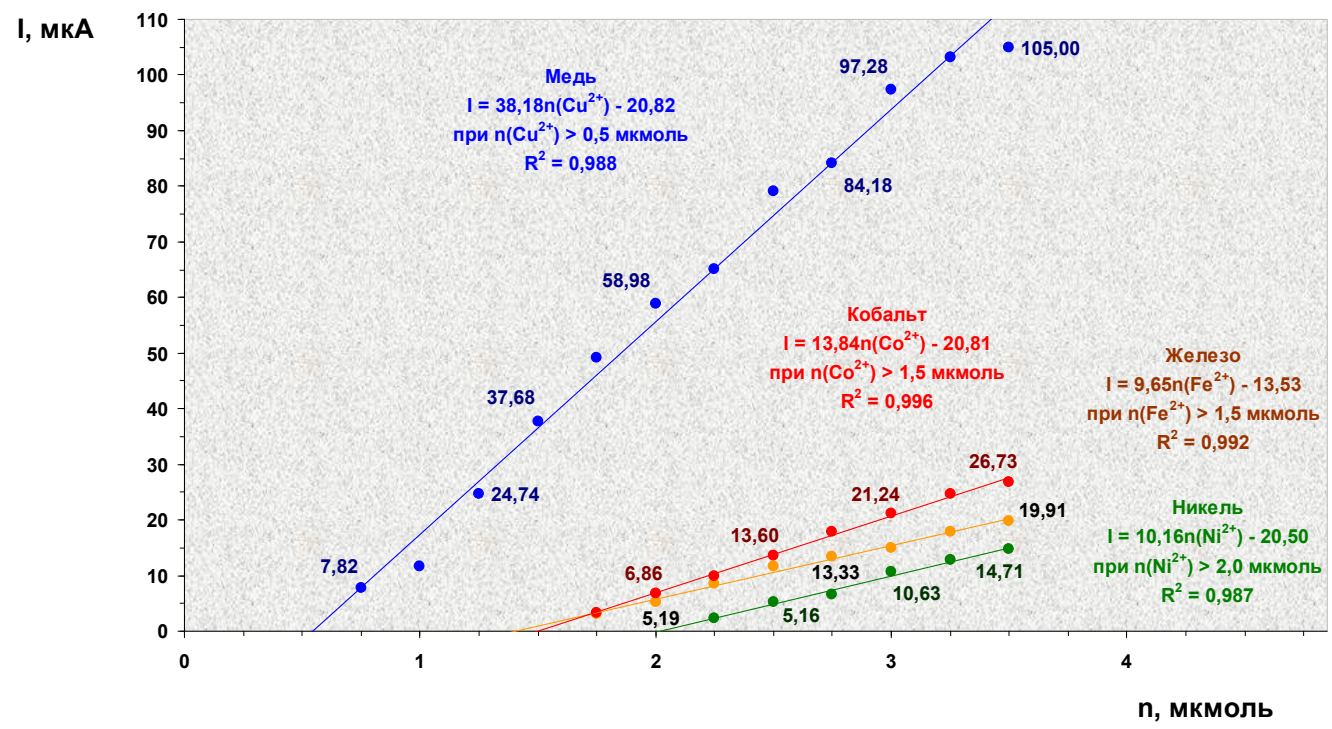

Рис. 2. Аппроксимация линейных зависимостей силы тока катодного восстановления гидроксильного радикала, I, от количества, n, ионов металлов разной природы 


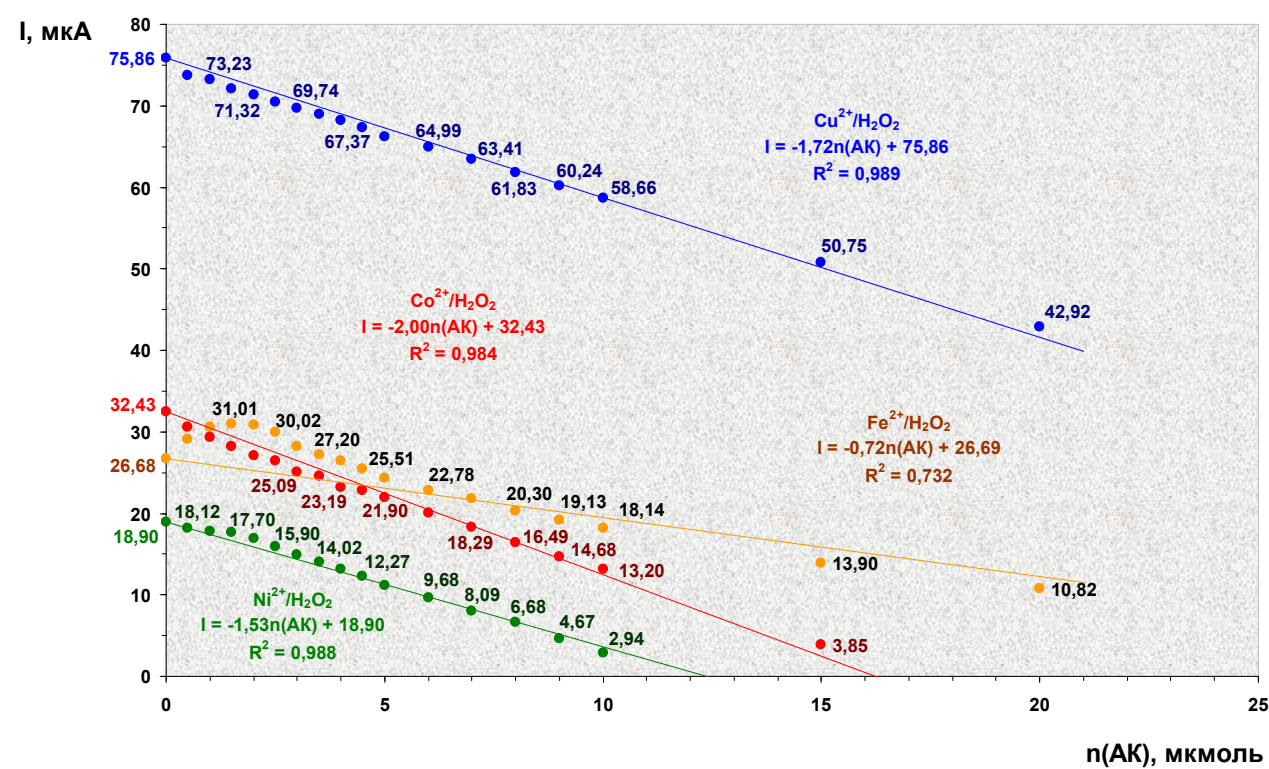

Рис. 3. Аппроксимация линейных зависимостей силы тока катодного восстановления гидроксильного радикала, I, от количества аскорбиновой кислоты, n(АК), в разных модельных системах

ет с количеством в системе ионов $\mathrm{Fe}^{2+}, \mathrm{Co}^{2+}, \mathrm{Ni}^{2+}$ или $\mathrm{Cu}^{2+}$, что подтверждается величинами достоверностей аппроксимации (значениями коэффициентов корреляции R). Для характеристики радикал-генерирующей способности, или прооксидантной активности (ПОА), ионов металла в данной аналитической системе мы использовали два параметра регрессионного уравнения: минимальное значение аргумента $\mathrm{n}$, при котором $\mathrm{I}>0$, и значение коэффициента регрессии. Физический смысл первого параметра: минимальное количество ионов металла, при котором образование радикала НО• сопровождается появлением соответствующего аналитического сигнала. Физический смысл коэффициента регрессии $k$ становится понятен из корреляционного уравнения. Общий вид уравнения

$$
\mathrm{I}_{\mathrm{i}}=k \mathrm{n}_{\mathrm{i}}-\mathrm{I}_{0},
$$

где $\mathrm{I}_{0}$ - рассчитанная координата точки пересечения аппроксимирующей линии с осью ординат в области воображаемых отрицательных значений І. Преобразуя уравнение, получаем

$$
k=\left(\mathrm{I}_{0}+\mathrm{I}_{\mathrm{i}}\right) / \mathrm{n}_{\mathrm{i}} .
$$

Таким образом, $k$ показывает аппроксимацию прироста сигнала НО• относительно количества добавленных ионов металла, выражается в мкА/мкмоль и является мерой ПОА. Среди исследованных ионов металлов ионы меди показывают наибольшую активность: дают минимальный сигнал радикала НО` при 0,75 мкмоль и его аппроксимирующий прирост ПОА $\left(\mathrm{Cu}^{2+}\right)=38,18$ мкА/мкмоль. Добавки ионов железа и кобальта сопровождаются появлением катодной волны гидроксильного радикала при 1,75 мкмоль, а никеля при 2,25 мкмоль, аппроксимация прироста в 3-4 раза меньше по сравнению с медью: ПОА $\left(\mathrm{Co}^{2+}\right)=13,84$ мкА/мкмоль, ПОА $\left(\mathrm{Ni}^{2+}\right)=10,16$ мкА/мкмоль, ПОА $\left(\mathrm{Fe}^{2+}\right)=9,65$ мкА/мкмоль. Высокая НО- 
генерирующая способность ионов меди подтверждается литературными данными. Согласно [7], константы скоростей реакций ионов меди с $\mathrm{H}_{2} \mathrm{O}_{2}$ на 3-4 порядка превышают значения констант скоростей аналогичных взаимодействий ионов железа с $\mathrm{H}_{2} \mathrm{O}_{2}$ классической реакции Фентона:

$$
\begin{aligned}
& \mathrm{Fe}^{2+}+\mathrm{H}_{2} \mathrm{O}_{2} \rightarrow \mathrm{Fe}^{3+}+\mathrm{HO}^{\cdot}+\mathrm{OH}^{-}\left(63-76 \text { л } \cdot \text { моль }{ }^{-1} \cdot \mathrm{c}^{-1}\right) \text { (реакция 1), } \\
& \mathrm{Cu}^{+}+\mathrm{H}_{2} \mathrm{O}_{2} \rightarrow \mathrm{Cu}^{2+}+\mathrm{HO}^{*}+\mathrm{OH}^{-}\left(10^{4} \text { л } \cdot \text { моль }^{-1} \cdot \mathrm{c}^{-1}\right) \text { (реакция 2), } \\
& \mathrm{Fe}^{3+}+\mathrm{H}_{2} \mathrm{O}_{2} \rightarrow \mathrm{Fe}^{2+}+\mathrm{HOO}^{-}+\mathrm{H}^{+}\left(10^{-3}-10^{-2} \text { л } \cdot \text { моль }{ }^{-1} \cdot \mathrm{c}^{-1}\right) \text { (реакция 3), } \\
& \mathrm{Cu}^{2+}+\mathrm{H}_{2} \mathrm{O}_{2} \rightarrow \mathrm{Cu}^{+}+\mathrm{HOO}^{\bullet}+\mathrm{H}^{+}\left(4,6 \cdot 10^{2} \text { л } \cdot \text { моль }^{-1} \cdot \mathrm{c}^{-1}\right) \text { (реакция 4). }
\end{aligned}
$$

Оценка антиоксидантной активности аскорбиновой кислоты. При построении (рис. 3) графиков зависимостей $\mathrm{I}=f(\mathrm{nAK})$ начала аппроксимирующих линий намеренно устанавливаются в экспериментальные точки $\mathrm{I}_{0}$ (значения на оси ординат выделены соответствующими цветами), измеренные при $\mathrm{n}(\mathrm{AK})=0$. Таким образом, корреляционное уравнение принимает общий вид

$$
\mathrm{I}_{\mathrm{i}}=-k^{\prime} \mathrm{n}(\mathrm{AK})_{\mathrm{i}}+\mathrm{I}_{0},
$$

откуда коэффициент $k^{\prime}=\left(\mathrm{I}_{0}-\mathrm{I}_{\mathrm{i}}\right) / \mathrm{n}(\mathrm{AK})_{\mathrm{i}}$, т.е. показывает аппроксимацию убыли сигнала гидроксильного радикала относительно количества добавленного антиоксиданта и является в данной системе мерой ингибирования модельного металл-индуцированного радикального стресса, или просто мерой антиоксидантной активности (АОА $=-k$, мкА/мкмоль).

Заметно (рис. 3), что начальные добавки аскорбиновой кислоты (0,50-1,50 мкмоль) в систему $\mathrm{Fe}^{2+} / \mathrm{H}_{2} \mathrm{O}_{2}$ сопровождаются не убылью, а приростом сигнала гидроксильного радикала $\left(\mathrm{I}_{\mathrm{i}}>\mathrm{I}_{0}\right)$. Последующие добавки до 4,00 мкмоль гасят избыток радикального стресса, индуцированный самой аскорбиновой кислотой, и только дальнейшие добавки укладываются в собственно антиоксидантную активность. Поэтому суммарная аппроксимация характеризуется сниженной достоверностью линейной корреляции $\left(\mathrm{R}^{2}=0,732\right)$ и минимальным по сравнению с другими системами значением модуля коэффициента регрессии, или наименьшей $\mathrm{AOA}_{\mathrm{AK}}\left(\mathrm{Fe}^{2+} / \mathrm{H}_{2} \mathrm{O}_{2}\right)=-0,72$ мкA/ мкмоль. Поведение сигнала I в системе $\mathrm{Fe}^{2+} / \mathrm{H}_{2} \mathrm{O}_{2}$ можно объяснить следующим образом. Скорость реакции окисленного железа $\left(\mathrm{Fe}^{3+}\right) \mathrm{c}_{2} \mathrm{O}_{2}$, регенерирующей $\mathrm{Fe}^{2+}$ (реакция 3), на несколько порядков меньше скорости реакции 1. Добавка 0,50-1,50 мкмоль восстановителя (аскорбиновой кислоты) в систему, содержащую 3,50 мкмоль ионов железа $\left(\mathrm{Fe}^{2+}+\mathrm{Fe}^{3+}\right)$, приводит к восстановлению $\mathrm{Fe}^{3+}+\mathrm{e}^{-} \rightarrow \mathrm{Fe}^{2+}$, ускоряя реакцию 1, что и проявляется приростом сигнала $\mathrm{HO}$. Подобные эффекты аскорбиновой кислоты известны как её прооксидантное действие, механизм которого служит предметом исследований научного сообщества. Так, согласно данным [9], прооксидантными свойствами обладают продукты превращений аскорбиновой кислоты в присутствии кислорода, а в отсутствие кислорода они не наблюдаются.

Полученные нами значения антиоксидантной активности аскорбиновой кислоты по ингибированию радикального стресса, провоцируемого ионами металлов разной природы, имеют существенные различия:

$$
\begin{aligned}
& \mathrm{AOA}_{\mathrm{AK}}\left(\mathrm{Co}^{2+} / \mathrm{H}_{2} \mathrm{O}_{2}\right)=-2,00 \text { мкА/мкмоль, } \mathrm{AOA}_{\mathrm{AK}}\left(\mathrm{Cu}^{2+} / \mathrm{H}_{2} \mathrm{O}_{2}\right)=-1,72 \text { мкА/мкмоль, } \\
& \mathrm{AOA}_{\mathrm{AK}}\left(\mathrm{Ni}^{2+} / \mathrm{H}_{2} \mathrm{O}_{2}\right)=-1,53 \text { мкА/мкмоль, } \mathrm{AOA}_{\mathrm{AK}}\left(\mathrm{Fe}^{2+} / \mathrm{H}_{2} \mathrm{O}_{2}\right)=-0,72 \text { мкА/мкмоль. }
\end{aligned}
$$

Причина различий, наиболее вероятно, заключается в индивидуальной кинетике взаимодействий аскорбиновой кислоты с ионами разных металлов, т.е. в разной степени проявления 
прооксидантного компонента активности. При анализе результатов для системы $\mathrm{Ni}^{2+} / \mathrm{H}_{2} \mathrm{O}_{2}$ (рис. 3) видно, что убыль сигнала НО` при $\mathrm{n}(\mathrm{AK})<2,00$ мкмоль меньше, чем при последующих добавках антиоксиданта, что можно расценивать как результат частичного вовлечения аскорбиновой кислоты в реакцию восстановления трёхвалентного никеля, аналогичную восстановлению трёхвалентного железа. Взаимодействие аскорбиновой кислоты с кобальтом, очевидно, минимально.

\section{Заключение}

При комбинировании модельной реакции Фентона или ей подобной $\left(\mathrm{Fe}^{2+} / \mathrm{H}_{2} \mathrm{O}_{2}, \mathrm{Co}^{2+} / \mathrm{H}_{2} \mathrm{O}_{2}\right.$, $\left.\mathrm{Ni}^{2+} / \mathrm{H}_{2} \mathrm{O}_{2}, \mathrm{Cu}^{2+} / \mathrm{H}_{2} \mathrm{O}_{2}\right)$ с вольтамперометрической регистрацией силы тока катодного восстановления гидроксильного радикала $\mathrm{HO}^{*}$ наблюдается линейная корреляция силы тока I, мкA, c количеством ионов металла в электрохимической ячейке, мкмоль. Регрессионный коэффициент уравнения линейной аппроксимации, показывающий прирост сигнала НО• относительно количества добавленных ионов металла, является мерой прооксидантной активности (ПОА) ионов металла. Показано, что ПОА максимальна у ионов меди (38,18 мкА/мкмоль) и уменьшается в ряду

$$
\Pi О А\left(\mathrm{Cu}^{2+}\right)>\Pi О A\left(\mathrm{Co}^{2+}\right)>\Pi O A\left(\mathrm{Ni}^{2+}\right)>\Pi O A\left(\mathrm{Fe}^{2+}\right)
$$

Аналогичный регрессионный коэффициент (со знаком минус) уравнения линейной аппроксимации, показывающий уменьшение высоты катодной волны НО' относительно количества добавленного антиоксиданта, является мерой ингибирования модельного металлиндуцированного радикального стресса, или антиоксидантной активности (AOA).

Аскорбиновая кислота показывает разное значение антиоксидантной активности в зависимости от природы провоцирующих стресс ионов металла, максимальное значение АОА $(-2,00$ мкА/мкмоль) наблюдается в системе $\mathrm{Co}^{2+} / \mathrm{H}_{2} \mathrm{O}_{2}$.

\section{Список литературы}

1. Free Radicals in Biology. Volumes I-VI. Edited by William A. Pryor. Academic Press, 19761984.

2. Halliwell B., Gutteridge J.M.C. The importance of free radicals and catalytic metal ions in human diseases. Molecular Aspects of Medicine 1985. Vol. 8(2). P. 89-193.

3. Halliwell B., Gutteridge J.M.C. Role of free radicals and catalytic metal ions in human disease: An overview. Methods in Enzymology 1990. Vol. 186. P. 1-85.

4. Jomova K., Valko M. Advances in metal-induced oxidative stress and human disease. Toxicology 2011. Vol. 283(2-3). P. 65-87.

5. Jomova K., Baros S., Valko M. Redox active metal-induced oxidative stress in biological systems. Transition Metal Chemistry 2012. Vol. 37(2). P. 127-134.

6. William A. Pryor. Free Radicals. McGraw-Hill, 1966. 354 p.

7. Bokare A.D., Choi W. Review of iron-free Fenton-like systems for activating $\mathrm{H}_{2} \mathrm{O}_{2}$ in advanced oxidation processes. Journal of Hazardous Materials 2014. Vol. 275. P. 121-135.

8. Патент 2426109 РФ. Лисецкий В.Н., Баталова В.Н., Лисецкая Т.А. Вольтамперометрический способ определения активности антиоксидантов. Опубл. 10.08.2011. [Patent 2426109 RU.

$$
-66-
$$


Lisetskij V.N., Batalova V.N., Lisetskaja T.A. Voltammetric method of determining activity of antioxidants. Publ. Date 10.08.2011 (In Russ.)]

9. Ivanova I.P., Trofimova S.V., Piskarev I.M. Evaluation of prooxidant properties of ascorbic acid. Biophysics 2013. Vol. 58(4). P. 453-456. 